Herrera-Morales, F. (2017). Familia y región: concepción y cambios de la familia en el Eje Cafetero. Revista de Antropología y Sociología: VIRAJES, 19 (2), 75-92. DOI: 10.17151/rasv.2017.19.2.5

\title{
FAMILIA Y REGIÓN: CONCEPCIÓN Y CAMBIOS DE LA FAMILIA EN EL EJE CAFETERO*
}

\author{
FABIAN HERRERA-MORALES**
}

Recibido: 1 de Abril 2017

Aprobado: 26 de Julio 2017

Artículo de Reflexión

\footnotetext{
* El presente artículo hace parte del avance del trabajo de investigación: "Familias y cotidianidad en la región del Eje Cafetero".

** Sociólogo, Magíster en Estudios de Familia y Desarrollo. Profesor del Departamento de Antropología y Sociología de la Universidad de Caldas. Manizales, Colombia. E-mail: fabian.herrera@ucaldas.edu.co. (1) ORCID: 0000-0002-1871-543X.
} 


\title{
Resumen
}

Objetivo. El artículoexpone un ejercicio de reconstrucción dela noción familiaregión Eje Cafetero a partir de los resultados de varios procesos investigativos; forjando como objetivo identificar la acepción cultural, los cambios y concepciones de la familia en la región. Metodología. En primer lugar se analiza la relación familiaregión desde aspectos simbólicos en la figura materna, la estructuración vincular y el hábitat; en segundo lugar se describe la muestra teórica más representativa de cambio en la familia del Eje Cafetero: la familia transnacional. Resultados y conclusiones. Finalmente se detalla, a nivel general, que las trasformaciones de la familia en la región se establecen por la incidencia de procesos de globalización en las formas de vida y las relaciones de autoridad entre padres e hijos; así como en las concepciones y expectativas de la reproducción familiar que, según los jóvenes y a pesar de los cambios, mantienen un hilo conductor que estrecha simbólicamente la relación familia-región.

Palabras clave: familia-región, región-madre, familia transnacional, globalización, nuevas generaciones.

\section{FAMILY AND REGION: CONCEPTION AND FAMILY CHANGES IN THE COFFEE TRIANGLE REGION}

\begin{abstract}
Objective: the article presents an exercise of reconstruction of the notion family- Coffee Triangle region from the result of various investigative processes, forging as an objective to identify the cultural meaning, the changes and conceptions of the family in the region. Methodology: In the first place, the family-region relationship is analyzed from symbolic aspects in the maternal figure, the linking structure, and the habitat. Secondly, the most representative theoretical sample of change in the family from the Coffee Triangle region, the transnational family, is described. Results and conclusions: Finally, it is detailed, at a general level, that the family transformations in the region are established by the incidence of globalization processes in the styles of life and in the authority relations of parents and children, as well as in the conceptions and expectations of family reproduction that, according to the young and despite the changes, maintain a common thread that narrows symbolically the family-region relationship.
\end{abstract}

Key words: Family-Region, Mother-Region, Transnational Family, Globalization, New Generations. 


\section{Introducción}

1 presente artículo consiste en un ejercicio de acercamiento a la constitución, cambios y transformaciones de la familia en el Eje Cafetero a partir de la relación familia-región expresada en simbolismos, significados y el papel de las nuevas generaciones en cuanto a las familias.

La familia en Colombia ha transitado por un largo camino en el que los cambios y transformaciones en su composición, organización y dinámica se han visibilizado poco a poco; su tránsito es inacabado, y varía según el contexto y la región en la cual se circunscriben las familias.

La familia como objeto de estudio y debate cobra relevancia en el marco de la interrelación multidimensional puesto que representa en la actualidad no solo aquel lugar de las emociones, lo íntimo, el mundo de lo privado, sino también una agencia que juega un significativo papel en la sociedad, el individuo y en instituciones como el Estado y la economía. Al ser la familia un eje de interrelación e interpretación social básico, se subraya que este no es el único marco referencial para el individuo; sin embargo, sí, es un arquetipo constituyente que en la relación individuosociedad forja el desarrollo humano y social; y por ello, un componente elemental que estructura los principales rasgos identitarios de una región y de un país.

La manera en la que se comprende la familia en interrelación con otras dimensiones de la sociedad se evidencia respecto a la transversalidad que tiene en innumerables problemáticas y fenómenos colectivos tales como los procesos de migración, urbanización, conflicto armado, desplazamiento forzado, aumento o reducción en las tasas de divorcios, establecimiento de nuevas unidades domésticas, aumento o reducción de la fecundidad, desempleo y pobreza. Las diversas situaciones se estructuran, y son estructuradas, en la diversidad de papeles y relaciones de poder en términos de género y generación. A su vez,

la familia se encuentra en los movimientos del propio proceso de individualización en contextos contemporáneos, en los cuales la reflexividad intrínseca conduce al yo a tomar su autonomía y libertad, desatarse de los anclajes y circular globalmente, produciendo la profunda tensión entre el proyecto familiar y el proyecto individual. (Palacio, 2010, p. 13)

Por consiguiente, las familias en general y las familias colombianas en particular no son ajenas a los inminentes cambios sociales (Palacio, 2009). 
Estas se organizan y se reorganizan de acuerdo a dichos fenómenos y a los procesos emergentes de la globalización con el surgimiento de nuevas formas de organizar familias; en especial, como una experiencia en el proceso de individualización (Herrera, 2015), la familia se encuentra en una constante transición.

No obstante, y pese a las fuertes transformaciones socioculturales que atraviesan tanto la familia como nuestras biografías en un mundo globalizado (Giddens, 2007; Therborn, 2007; Arriagada, 2009; Jelin, 2010), hay que resaltar que los cambios también se generan con incidencia en las particularidades de cada región; para el contexto que traemos entre manos, la genealogía familiar y la idiosincrasia conllevan a la conservación de creencias y prácticas relacionales y de organización que se resisten a cambios abruptos o definitivos. A pesar de los radicales cambios, la sociedad colombiana sigue siendo una sociedad con fuerte tendencia familística (Puyana, 2012; Guzmán y Chaparro, 2013; Flórez y Sánchez, 2013); por ello hay que resaltar que la familia colombiana, especialmente la de la región del Eje Cafetero, comprende y acepta como ideal a la familia tradicional (Pachón, 2007; Ministerio de Cultura, 2011; Curiel, 2013); aspectos que con la época, pero también de acuerdo a la clase social y al nivel educativo, van reconfigurándose para dar paso a la organización de nuevas formas familiares; aunque se destaca a la familia nuclear como la principal, mas no la única.

Para llegar a un acercamiento de la concepción de la constitución y cambios de la familia en el Eje Cafetero es relevante la mirada generacional, teniendo en cuenta cuáles son las tendencias - y marco de significados- y el papel que tienen los jóvenes en la resolución de los aspectos tradicionales que establecen las lógicas familiares en la región respecto a las más pronunciadas transiciones que vive la familia en un mundo de inevitables procesos de globalización.

\section{La familia en el Eje Cafetero: asunto de reflexión desde una investigación clásica}

Al tratar sobre la familia en la región del Eje Cafetero es básico resaltar estudios clásicos como los realizados por la investigadora social Virginia Gutiérrez de Pineda, quien en la década de los años 60 y 70 llevó a cabo uno de los estudios más completos sobre la complejidad de la familia en Colombia, logrando hacer un acercamiento significativo en la relación familia-región. 
La autora de libros como La familia en Colombia: trasfondo histórico (1964), La Familia y cultura en Colombia: tipologías, funciones, y dinámica de la familia (1968) y Estructura, función y cambio de la familia en Colombia (1973), resalta que las regiones en Colombia funcionan como "complejos culturales". Es decir que la región no se reduce solo a sus condiciones geológicas y geográficas, sino que allí se establecen unos patrones culturales transversalizados por el hábitat que inciden en las cualidades antropológicas y sociológicas de cada población. En esta perspectiva se comprende la región como un aspecto significativamente influyente en las regularidades culturales que estructuran las formas de vida social, las organizaciones familiares y las relaciones de género y generación de cada población.

En relación con lo anterior hay que resaltar que, en las décadas en las que la autora realizo la investigación, el país se encontraba en fuertes procesos de urbanización y migración interna; aspectos que conllevan a que sea tan difícil, hoy por hoy, determinar una identidad sumamente marcada por cada región; no obstante, lo que sí podemos establecer es un acercamiento a los rasgos regionalmente más sobresalientes sobre la constitución de las familias.

A nivel del territorio nacional, Virginia Gutiérrez de Pineda identifica cuatro regiones: la región andina, la región de los santanderes, la región de la montaña y la región del complejo litoral minero. Cada una con sus especificidades. Aquí, se considerará una: la región de la montaña.

En el caso de la región del Eje Cafetero cabe resaltar que según los resultados de sus investigaciones hace parte del complejo cultural de la montaña, también denominado antioqueño, que en términos de su sistema cultural expresa determinadas simbologías.

La institución religiosa alcanza en este complejo su plenitud máxima, como posición en la sociedad y proyección ética sobre el individuo y la estructuración familiar. La unidad doméstica se configura sobre base del matrimonio, siendo este complejo el que suministra los más altos porcentajes de nupcialidad. La familia presenta un marcado cuadro matriarcal, y fuertes nexos familiares en la unidad extensa unilineal uterina. (Gutiérrez de Pineda, 1994, p. 33)

El complejo cultural de la montaña constituye un fuerte marcador de identidad, (Sandoval y Moreno, 2008) "factiblemente el más taxativo"; una identidad unida a la aquiescencia de la moral cristiana (Zuleta, 2010), reflejada en las pautas de comportamiento individual y colectivo.

Por un lado, si bien los procesos de secularización han sido fuertes y 
permanentes en las últimas décadas en Colombia, se resalta que las familias de la región del Eje Cafetero están incididas por la religiosidad cristiana la cual ya sea por filiación o práctica logra una influencia determinante en la configuración de valores, creencias, proyección ética y moral en la vida cotidiana; y, por otro, si bien hay unidades domésticas que se constituyen a partir del matrimonio, se resalta la alta tendencia de uniones libres o familias de hecho desde la segunda mitad del siglo XX (Silva y González, 2007); no solo en la región, sino en el resto del país ${ }^{1}$.

Una de las pautas culturales que se infiere de la estructura familiar tiene que ver con la marcada tendencia matriarcal. Es decir que en la organización familiar se establece un predominio en el significado del papel de la madre, la familia de origen de la madre en los hijos y su gobernabilidad del hogar, sin invisibilizar el papel del padre quien establece su autoridad. De acuerdo a la investigación de Corella (2012), sobre la estructuración de la autoridad en un grupo de familias de un sector popular de la ciudad de Manizales,

la autoridad y la decisión de distribuir los ingresos al interior de la familia continúan recayendo en el hombre. Haciendo visible el significado que tiene el recurso de género para el ejercicio de la autoridad al interior de la familia, basado en una concepción cultural y tradicional de las funciones que tienen el hombre y la mujer al interior del grupo familiar. Esta pauta tradicional y cultural genera que las relaciones de poder que se establecen entre los miembros de la familia contienen elementos disimétricos que resultan en situaciones donde se da prioridad al papel de algunos miembros en las decisiones familiares, como es el caso de la decisión en la distribución de los recursos económicos al interior de la familia. (p. 118)

En otros términos, se determina la separación entre lo público y lo privado como una cuestión de género y generación donde el papel de la mujer y los hijos se concibe al interior del hogar (lo privado) y el papel del hombre con relación a las tomas de decisiones económicas con autoridad en el sistema de permisos y castigos en la familia (lo público). Esta figura, sin embargo, no se presenta en todas las familias: si bien es una tendencia patriarcal importante porque se sigue reproduciendo, también hay que reconocer que se han forjado rupturas como cuando el poder del padre en la familia cambia debido a que los ingresos monetarios no recaen en él sino en la mujer; o en el papel de los hijos, como veremos más adelante,

\footnotetext{
${ }^{1}$ En la investigación realizada por la organización global Child Trends (con datos de PROFAMILIA y el DANE en este caso) Colombia sobresale por ser el país con mayor porcentaje de cohabitación o uniones libres. El 39 \% de las parejas entre 18 y 49 años de edad vive de esa manera (El Tiempo, 2013).
} 
especialmente aquellos que vienen prologando el ideario de establecer una nueva familia, reafirmando su estancia en la casa de origen por más tiempo; por tanto, la asimetría de las relaciones de autoridad suele transformarse cuando la conyugue o sus hijos se convierten en los proveedores económicos.

\title{
Familia y región: la simbología de la madre
}

Al hacer una lectura contemporánea de los estudios de Virginia Gutiérrez de Pineda podemos encontrar que el lugar de la madre en las familias del Eje Cafetero representa un lugar simbólico en el sentido del arraigo, el amor y el cuidado; y que a pesar de que son categorías compartidas por el sistema patriarcal (Fraser, 2015; Mateos, 2013) estas no se comprenden solo en términos de una sumisión sino en términos de la sacralidad y espiritualidad de su representación (Cohen, 2014), imaginario que se constituye en las lógicas del sistema de valores y de creencias de la familia.

\begin{abstract}
Volvemos al recuerdo, a la madre, a la que engendró el progreso, a la que se le debe el agradecimiento, el sustento, a la benefactora, depositaria de coplas y canciones, poemas, pinturas y evocaciones del paisaje que cobija la vida. El Eje Cafetero también actúa como la madre tierra en el imaginario, que vuelca las evocaciones al ser original. Este ser femenino, fértil, convoca a un mundo de relaciones más abierto, está asociado al vitalismo, a la fuerza generatriz que conlleva por su naturaleza de eterno recomenzar a una ecología de espíritu y a la vez a una ecología del mundo. (Robledo, 2008, p. 212)
\end{abstract}

De acuerdo a la investigación de Robledo (2008) hay una relación entre la región y el marco de la memoria puesto que sus habitantes suelen manifestar como expresión de herencia su apego al territorio, como representación de la madre, en la acentuación de una región-madre. En este sentido el papel de la madre prevalece en la impronta de la génesis familiar y del pueblo al representar la transmisión y la renovación de afectos y valores. Simbólicamente la familia se establece en el imaginario colectivo como la agencia que dimensiona la integridad subjetiva, emocional y vincular del ser humano.

Por tal motivo, se resalta que, en la vida práctica, la familia como institución para la procreación se ha transformado progresivamente en el sentido de que las nuevas generaciones no ven en ella la única vía de realización personal tal como pensaban los antepasados; es así, que es factible la conservación del simbolismo sin tender a una radical reproducción de la familia en la proyección real de la vida. 


\section{La familia transnacional como tendencia de cambio de la familia en el Eje Cafetero}

Así como la representación simbólica de la madre se vincula con el origen, el arraigo, el apego, la región del Eje Cafetero representa un marco de sentimientos similar para aquellos — miembros de familia o familias enteras - que hacen parte de las generaciones que empezaron el proceso migratorio; que al día de hoy constituyen no solo una realidad, sino un eje identitario en el que se ha hecho normal que las familias de la región -al menos en la mayoría - tengan un familiar que migró hacia el exterior y cuya particularidad se resalta en los fuertes lazos (González y Restrepo, 2010) que unen una familia entre sí y su región: la región de origen o procedencia. “Los departamentos de Antioquia y del Eje cafetero (Caldas, Quindío y Risaralda) presentan uno de los índices más altos del país, convirtiéndose en un determinante para las nuevas dinámicas familiares" (Restrepo, 2016, p. 128).

Para comprender el proceso de migración, que ha generado el desarraigo con la región-madre, hay que tener en cuenta tres causas del contexto- que surgen como momentos de ruptura que llevaron a que la migración internacional se estableciera como una de las mayores tendencias de cambio en la familia de la región, siendo la primera el choque de tiempos adversos como los acecidos como consecuencia del terremoto de 1999; la segunda causa, la recesión económica en el mismo año y por lo tanto la subida del índice de desempleo; finalmente, la tercera, la crisis de la economía cafetera (López y Loaiza, 2009).

Las nuevas realidades que crea la migración en la familia se establecen en la concepción de familia transnacional, entendida esta como "una familia que tiene miembros de parentesco inmediato en varios países simultáneamente, que mantiene comunicación y sentido de unidad familiar, que tiene intercambio de bienes y servicios y una circularidad de presencia física, aunque esta no sea frecuente" (Caballeros, 2010, p. 4). "Se refiere entonces al hecho que uno o varios miembros se encuentran viviendo físicamente en otros países y que la familia debe mantenerse a través de las fronteras" (Zapata, 2009, p. 1753); es decir que la familia transnacional llega a establecerse como un "espacio familiar y de vínculos afectivos de confianza en un contexto en el que las conexiones están geográficamente dispersas" (Parella, 2007, p. 152).

Con base en ello se trata de una nueva noción de familia que genera una secuencia de cambios sociales tanto para esta como para sus miembros, pues los migrantes al mantener una comunicación y un intercambio de bienes y servicios causan una serie de transformaciones en sus dinámicas ya sea en el espacio familiar, vínculos afectivos o en el contexto en donde 
la familia de origen continúa viviendo. Teniendo en cuenta que su diario vivir cambia por la migración de uno o varios miembros y haciendo que su estructura familiar se modifique al percibir como las relaciones familiares, la comunicación, la socialización, funciones y responsabilidades de los integrantes obligan a la familia a reestructurar sus rutinas y a la vez desarrollar capacidades para comunicarse y aprender a vivir con la distancia que deja la migración; poniendo de relieve las oportunidades que esta promueve dentro de un contexto económico en la familia, lo que genera nuevos ingresos para mejorar la calidad de vida.

“El Eje Cafetero es una de las zonas con mayor número de personas emigradas en relación con su población" (López y Loaiza, 2009, p. 7). En este sentido la economía del Eje Cafetero afecta las pocas posibilidades que las familias tienen de alcanzar los objetivos propuestos y aquello que han idealizado como "calidad de vida", en donde la economía influye en los recursos necesarios para alcanzar a suplir por lo menos las necesidades básicas. Por lo tanto la migración no ocurre de manera simple puesto que entre el contexto migratorio y las características de las familias se constituyen múltiples situaciones y relaciones que confluyen para mantener el equilibrio ante la separación y la garantía de ajustes que promuevan, a pesar de la distancia, un mejoramiento en el desarrollo social y humano de los integrantes.

Entre las motivaciones más fuertes de los migrantes se establece el factor económico (Ciurlo, 2014), referido a la búsqueda de otras opciones de empleabilidad que le permitan lograr mayores ingresos y garantizar así una mejor educación, salud y calidad de vida para la familia. Es así como se evidencia que los padres o hijos que viven la experiencia migratoria están en el deber de mejorar la calidad de vida de la familia como respuesta a la coyuntura y al conjunto de problemáticas socioeconómicas que afrontan en la región. Aunque el papel de los padres y madres migrantes no solo se enfoca en un mejoramiento del bienestar económico de la familia sino también al cuidado (Degrave, 2007), expresado en la práctica y aporte al desarrollo humano y familiar donde el conjunto de experiencias, motivaciones y expectativas se orientan al despliegue de mayores oportunidades culturales y económicas de los integrantes de la familia, encontrando aspectos que ayudan a su progreso.

Si nos preguntamos acerca de las relaciones familiares en la cotidianidad de las familias transnacionales hay que resaltar que cuando los padres emigran es usual que los hijos pasen a ser cuidados por otras personas (Soto, 2011; Restrepo, 2016), en la mayoría de los casos por las abuelas maternas; aunque la autoridad pasa a los cuidadores, no se elimina por completo la toma de decisiones por parte de los padres migrantes. 
Es decir que, en la familia transnacional se asumen nuevos roles ya sea el hermano mayor cuidando al menor o la tía o abuela cuidando a los menores, surge una variabilidad en la figura de autoridad en los padres a distancia. Asimismo, el establecimiento de vínculos se reconstruye a través del uso de las nuevas tecnologías para mantener las relaciones socioafectivas mediante la comunicación con los padres o la relación madre, hijo u otro integrante que haya emigrado.

Por otra parte, el mejoramiento de las condiciones económicas permite a las familias del Eje Cafetero forjar las expectativas de una vivienda propia o incluso un negocio o capital financiero con la promesa de regresar algún día y poder establecer una inversión que genere ingresos más estables.

\section{La familia y sus cambios desde el papel de los jóvenes en el Eje Cafetero}

Es importante tener en cuenta que la familia del Eje Cafetero no escapa a las nuevas formas de estructuración organizacional y de vinculación que tienen relación con la modernización y la Modernidad (Arriagada, 2009) tales como las nuevas pautas de consumo y de trabajo (modernización), la autoridad al interior de la familia y la promoción de la libertad social e individual (Modernidad). Condiciones globales que asientan determinadas particularidades en la medida en que la percepción y las relaciones con el ámbito familiar varían de acuerdo a las situaciones socioculturales de cada región.

Al día de hoy, las nuevas pautas de consumo y de trabajo han redimensionado las relaciones familiares y el papel de los hijos en las familias; pues los jóvenes junto con sus familias de origen están en la permanente tendencia a aumentar los índices de consumo tanto en bienes y servicios como en la accesibilidad a ciertos lujos; considerando, además, que las nuevas generaciones fijan una mayor permanencia en la casa de sus padres por la inestabilidad que ofrece el mercado laboral y por la dificultad de acceder a una nueva casa o sostener una nueva familia debido a que los ingresos monetarios cada vez más se estiman suficientes solo para las necesidades de vida actual que estos tienen transitoriamente.

Los padres se encargan de gastos más "primarios" como aspectos básicos de la canasta familiar y pago de cuentas de agua y energía eléctrica, mientras a los jóvenes les suele corresponder otros gastos más "secundarios" como puede ser el pago de telefonía e Internet o la compra de nuevos alimentos que no eran incluidos en la canasta familiar. (Rojas, 2013, p. 70) 
Esto genera que los jóvenes tengan una libertad con respecto a la optimización de sus recursos, invirtiendo en otras actividades de consumo y teniendo así una "autonomía relativa".

Según Rojas (2013) los jóvenes se van posicionando en el espacio de las relaciones familiares a partir de la adquisición de nuevas responsabilidades económicas, lo que va posibilitando tener voz y voto en la toma de decisiones en su vida familiar y en su vida personal.

Esta toma de decisión sobre su vida personal y familiar es una práctica que incentiva a la democratización de las relaciones familiares y a la transformación de la noción de autoridad; pues se resalta que la participación en las relaciones de autoridad recae en aquellos que proveen aportes económicos al hogar, ya que estos tienen no solo la oportunidad de opinar sino de tomar decisiones frente a la variedad de situaciones que vive la familia.

Por otro lado, también suelen haber tendencias donde los padres se encargan de las decisiones familiares y los jóvenes de las decisiones más personales. Sin embargo cuando es una decisión que los involucra a todos, hay un consenso que establece una democratización de las relaciones.

La promoción de la libertad social e individual al interior de la familia, genera transformaciones en la intimidad y en la sexualidad de los jóvenes cuando estos viven con su familia de origen. Ya los padres toleran las relaciones prematrimoniales amorosas o sexuales que estos tienen por fuera del hogar, por lo que "se hace 'razonable' el permanecer con sus padres dado que cuentan con la autonomía para decidir sobre sus aspectos más íntimos y personales" (Rojas, 2013, p. 70).

Otra dimensión a resaltar, tiene que ver con las expectativas de los jóvenes sobre sus proyectos personales de matrimonio y de familia. Para Duque (2012), respecto a la proyección a futuro que tienen los jóvenes, las expectativas de casarse y de tener una nueva familia como asunto primario suelen variar de acuerdo a las situaciones particulares tales como la clase social (Tiramonti, 2006) y el nivel educativo. En el caso del Eje Cafetero, especialmente en los jóvenes de clase media, tiende en buena medida a ser un proyecto secundario porque como proyecto primario se establece la necesidad de continuar una formación académica y profesional, así como la urgencia de buscar un empleo que brinde mejor estabilidad económica. Se resalta, entonces, que los jóvenes no proyectan la idea de matrimonio o de familia sin previamente forjarse una profesión y un empleo que responda a la expectativa de una mejor calidad de vida. En otras palabras los jóvenes tienden a preferir vivir con su familia de origen hasta nuevo aviso puesto que la casa de origen promete un soporte y amparo, permitiendo a los jóvenes tener una liviana responsabilidad en la que pueden tomar sus 
propias decisiones; sin embargo, cuando esta posibilidad es vulnerada, los jóvenes proyectan vivir separados de su hogar de origen.

Caso contrario sucede en el caso de los jóvenes rurales del Eje Cafetero que tienen mayores expectativas de migrar a la ciudad bien sea a estudiar, trabajar o residir. La incidencia del modelo económico y de desarrollo social derivada de los procesos de globalización, genera que en los jóvenes rurales se despierten aspiraciones desde muy temprano ajenas al campo; lo anterior, los lleva a dejarlo y a migrar a la ciudad en búsqueda de la idea de una mejor calidad de vida. Sin embargo, de acuerdo a una investigación de Jurado y Tobasura (2012), pese a que los jóvenes aspiran a vivir en la ciudad, reconocen que la vida en el campo proporciona un desarrollo humano y social puesto que la tranquilidad y la posibilidad de una vida más saludable y austera se encuentran allí; aunque también detallan que no es un sector valorado en el país, por lo que las actividades económicas realizadas no son lo suficientemente bien pagas que otras labores que puedan emprender en la ciudad. Esto lleva, a que los jóvenes expresen crisis de identidad y resignificaciones en sus proyectos de vida.

\section{Nuevas generaciones, individualización y concepciones de familia en el Eje Cafetero}

Los jóvenes a través del acompañamiento de sus familias construyen un significado a su vida tanto individual como familiar, generando con ello una perspectiva y expectativa frente a la forma en la cual desean construir o no sus propias familias.

Los grupos familiares se reconocen a través de su labor socializadora y también como escenario en el que confluye el aprendizaje de formas de actuar, ser y ver el mundo que responden a tradiciones y un equipaje cultural compuesto por el reconocimiento y la capacidad de los individuos de tomar decisiones, modificar y construir su identidad. Vislumbra las motivaciones por las que los jóvenes poco a poco se alejan de la forma en la que su familia de origen fue constituida, respondiendo necesariamente al proceso de individuación por medio del cual los individuos de manera autónoma buscan y eligen la forma de vivir su vida. En este sentido se hace necesario retomar los postulados de Jelin (2010):

la individuación incluye el reconocimiento de la necesidad de observar nuestras vidas y acciones desde nuestro propio punto de vista. Esto implica el surgimiento de la autonomía personal, en el sentido de la capacidad de tomar decisiones propias, basadas en la información y 
en el conocimiento, en conjunto con el reconocimiento de los propios deseos. (p. 34)

Si bien los sujetos construyen su identidad en relación con sus familias, su grupo de pares y en general con todos los escenarios en los que interactúa, su configuración y su construcción no están determinadas en su totalidad ni sujetas a estructuras familiares ni sociales; ya que los sujetos a medida que avanzan en su proceso de individuación se convierten en actores protagónicos de su vida al permitir el reconocimiento de derechos y formas alternas de ser, actuar, pensar, de verse y ver a los otros, posibilitando el proceso de reflexión sobre su realidad y lograr así transformarla.

Sobre las expectativas de pareja de los jóvenes universitarios del Eje Cafetero, Duque (2012) encuentra que las aspiraciones de los jóvenes están orientadas hacia una estabilidad emocional; de ahí la idea de pensar en una nueva familia. Sin embargo es una proyección a futuro, ya que estos sugieren que antes se debe alcanzar una formación académica y profesional definida o una cierta estabilidad económica. En este sentido los jóvenes especialmente de clases medias y altas, deniegan la posibilidad de una familia hasta no lograr una mejor calidad de vida personal.

Tiramonti (2006) expone que en el tránsito por los procesos de individualización prevalece la exigencia de "ser sí mismo"; en donde las personas abordan esta experiencia desde condicionamientos sociales diferentes que les proveen recursos, inhibiciones, habilitaciones, expectativas y miedos que se hacen presentes en los modos de abordar proyectos a futuro. En la investigación citada la autora explica como al día de hoy existe una experiencia de desinstitucionalización y una corrosión de las formas de organización social tradicionales como la familia, el Estado y la religión. Teniendo el mayor peso los jóvenes de sectores populares quienes sometidos a la tiranía de la necesidad y a la amenaza de la desintegración familiar, en tanto que es un lugar donde no es posible el desarrollo de sus expectativas personales, son llevados con urgencia a entrar en las lógicas de la individualización contemporáneas y buscar así la manera de trazar sus logros individuales. Situación contraria con los jóvenes de clases medias $\mathrm{y}$ altas donde las manifestaciones de individualización son trazadas por factores culturales diferentes. No obstante tanto para las clases populares como para las clases medias y altas la educación sigue siendo no solo un factor de movilidad social, sino un condicionante frente a los proyectos individualizadores como el hecho de vivir solos y proyectar una vida individual.

De acuerdo con Yopo (2013) los procesos de individualización están siendo utilizados para describir y analizar las transformaciones culturales 
de la familia, las relaciones con la intimidad, el consumo cultural, el género, la política y de una forma particular en la organización y producción de nuevas subjetividades.

Villegas (2006) señala que la individualización ha sido posible por el surgimiento de amplios círculos de acción social propiciada por los cambios de las estructuras sociales y económicas de hoy; asimismo, el individualismo surge como una condición contemporánea de la familia y como noción opuesta al individualismo Homo economicus generalmente conocido como una forma de narcicismo y egoísmo con altos intereses especialmente económicos. Por tal motivo el investigador destaca que el individualismo como distinción de singularidad, y a la par como nueva forma de organización social, establece tanto la redistribución de roles en el trabajo doméstico como nuevas formas de relación e integración social.

En otros términos la decisión de los jóvenes se apoya en el propósito de construir familias como una organización diversa que responda a sus necesidades, intereses, deseos y motivaciones dentro de los que se destaca construir una familia propia sin que ello opaque la necesidad de sentirse autónomos y libres.

Es así como se entiende que el proceso de individualización y las nuevas organizaciones familiares están en constante interacción; los sujetos, a medida que construyen y reconocen sus libertades y autonomía, van visualizando la necesidad de resignificar la forma y el significado de familia. Permitiendo identificar motivaciones que llevan a construir nuevas familias donde las nuevas co-residencias no están orientadas a sacrificar los intereses laborales y/o educativos, libertades y demás, y donde la elección opcional de concebir hijos deja de ser un objetivo de la pareja y se convierte en una decisión de tendencia democrática.

Estas razones, y muchas otras, son las que motivan a construir nuevos hogares que toman fuerza entre los jóvenes como forjar vínculos a modo de Living Apart Together': parejas estables que comparten gran parte de su tiempo, pero que no cohabitan permanentemente; muchos de ellos aún viven con sus familias de origen o algunos poseen viviendas unipersonales.

El anterior ejemplo hace parte de un sinfín de organizaciones familiares que dejan atrás la forma tradicional de familia, posicionando la diversidad familiar como una realidad cada vez más grande y real. En este sentido se reconoce el interés por asumir nuevas formas de vida en familia, de reconocerse y de reconocer al otro, que van posicionando relaciones más equitativas, solidarias e iguales. En relación con la última premisa, se trae a

\footnotetext{
2 "Estar juntos, pero separados". "Los jóvenes que siguen residiendo en el hogar familiar, como medio de defender su independencia personal y mantienen simultáneamente relaciones de pareja estables y duraderas" (Alberdi, Flaquer e Iglesias, 1994, p. ).
} 
escena un aporte de Di Marco (2005) donde se hace evidente contemplar el papel de la mujer y cómo a través de este se posibilita el logro de relaciones democráticas al interior de la organización familiar.

Las familias cambian y también se reacomodan y sobreviven a los cambios, denotando en su interior nuevos perfiles y dinámicas. Hoy por hoy, incluso con todas las alteraciones que esta institución está atravesando. Uno de los cambios más importantes que están atravesando las familias se relaciona con la creciente incorporación de las mujeres al empleo remunerado. La importante afluencia femenina en el espacio público redefine el marco de las relaciones en el espacio privado. Y esta redefinición no necesariamente implica un déficit en las familias, sino que, por el contrario, puede contribuir a la construcción de relaciones más democráticas entre hombres y mujeres y entre adultos y niños. (Di Marco, 2005, p. 7)

Es así como se reconoce el camino que han atravesado las familias y los grandes cambios que han sufrido, lo que ha posibilitado la capacidad para incidir en su propia realidad a través de la concienciación de la misma y de los aspectos que rodean sus dinámicas, estructuras, formas de comunicarse y de relacionarse con el propósito de develar aspectos que afectan su desarrollo (encuentros y desencuentros, desigualdades e inequidades) y visibilizar aquellas habilidades y capacidades que les permitan potenciar procesos democráticos.

Para finalizar, se expone la siguiente afirmación de Giddens (2000):

de todos los cambios que ocurren en el mundo, ninguno supera en importancia a los que tienen lugar en nuestra vida privada - en la sexualidad, las relaciones, el matrimonio y la familia-. Hay en marcha una revolución mundial sobre cómo nos concebimos a nosotros mismos y cómo formamos lazos y relaciones con los demás. (p. 65)

Avanzar en estudios de la familia, su transformación y nuevas formas de organización, requiere del reconocimiento de esta con un amplio potencial de cambio para construir miradas alternas frente a su papel a nivel individual y social; pues, si bien la familia es receptora de transiciones y transformaciones sociales, construye e influye de manera profunda en cambios a nivel meso y macro. Lo que conlleva a que las familias regionales inevitablemente entren en las dinámicas de cambios globales.

Para el caso singular del Eje Cafetero, la familia ha vivido cambios derivados de los procesos de globalización. Sin embargo, dichos cambios devienen y se combinan con formas tradicionales en las que se estructuran los vínculos y la organización familiar. 


\section{A modo de conclusión}

La familia en la región del Eje Cafetero no escapa a los procesos de globalización e individualización mundiales; expresión de ello, son las relaciones familiares y expectativas de familia en las nuevas generaciones regionales. Sin embargo estos cambios han logrado entrelazarse con los códigos culturales y representaciones simbólicas que aún se conservan tales como el fuerte sentido familístico, comunitario, solidario y el sentimiento de arraigo al hogar; siendo este, un sentimiento y territorio de la intimidad.

Dimensión del sentimiento y la identidad que vive en tránsito puesto que la realidad familiar en la región también se ha estructurado a causa de los estragos de las fuertes condiciones políticas, sociales y económicas de nuestros tiempos: desde el conflicto armado, el desarraigo, el desplazamiento del campo a la ciudad hasta el asedio de una globalización negativa y su incidencia generada por otras violencias de carácter económico como el desempleo, la carestía en la canasta familiar, la ruptura de vínculos, en la subvaloración de los lazos ancestrales y lo fraternal; expresiones enmarcadas en la violencia familiar, sexual y de género. Problemáticas que reivindican la importancia de las políticas públicas de familia y un mayor compromiso institucional en los diversos hogares de la región y el país.

Al señalar que la familia del Eje Cafetero no escapa a los álgidos procesos de la globalización y condiciones estructurales del país, cabe enmarcar que dichos cambios no son en su totalidad negativos debido a que también se forjan transformaciones constructivas que hacen llamados continuos a la democratización de los procesos familiares, de sus vínculos, y a la aceptación de las nuevas relaciones socioafectivas y tendencias familiares. Vivimos otros tiempos llenos de lenguajes que no olvidan sus orígenes, pero que no se resisten del todo a las emergentes configuraciones emocionales.

La laboriosidad como un valor ancestral en el marco de las familias del Eje Cafetero se reivindica a través de la urgencia del trabajo comunicativo al interior de las familias y las relaciones socioafectivas como canalización constitutiva de pensar y aceptar la diversidad familiar y subjetiva como figura que representa su paisaje social. Siendo posible en el marco educativo de los jóvenes el aporte cultural y de conocimiento de los padres, madres e hijos y su experiencia de vida en países tal vez más democráticos como es el caso de las familias transnacionales; y finalmente en una mayor urgencia del papel participativo y garantías de derechos por parte del cuadro político y social del Estado y sus instituciones. Es así que, con un mayor ejercicio democrático al interior de las relaciones familiares y de las familias con las demás agencias de la sociedad, es que se contribuye al posconflicto; al ser 
las familias de la región Eje Cafetero forjadoras de paz y constructoras de país.

\section{Referencias bibliográficas}

Arriagada, I. (2009). La diversidad y desigualdad de las familias latinoamericanas. Recuperado de http://www.ucaldas.edu.co/docs/seminario_familia/PONENCIA_IRMA_ ARRIAGADA.pdf.

Caballeros, A. (2010). Guatemala: migración, mujeres y familias: el costo humano y social de la migración internacional. Recuperado de http://www.gloobal.net/iepala/gloobal/fichas/ ficha.php?entidad=Textos\&id=10522.

Ciurlo, A. (2014). Género y familia transnacional. Un enfoque teórico para aproximarse a los estudios migratorios. Revista Científica general José María Córdova, 12 (13), 127-161.

Cohen, M.C. (2014). El despertar de lo sagrado femenino. Yachay-Kusunchi, 2 (1), 15-19.

Corella, L.C. (2012). La estructuración de la autoridad a partir de los recursos que poseen los agentes: el caso de las familias de la comuna San José. Manizales, Colombia: Universidad de Caldas.

Curiel, O. (2013). La Nación heterosexual: análisis del discurso jurídico y el régimen heterosexual desde la antropología de la dominación. Bogotá, Colombia: Brecha Lésbica y en la frontera.

Degrave, F. (2007). Las fronteras del cuidado. Reflexiones para una conceptualización del cuidado a las personas de edad dependientes a partir de un estudio de caso en Bruselas. En Herrera, I.Y. (ed.), Nuevas migraciones latinoamericanas a Europa. Balances y desafíos. Quito, Ecuador: FLACSO.

Di Marco, G. (2005). Democratización de las familias: estrategias y alternativas para la implementación de programas sociales. Buenos Aires, Argentina: UNICEF.

Duque, A.L. (2012). Un abordaje sociológico de las expectativas en las parejas heterosexuales: el caso de los jóvenes universitarios de 18 a 26 años estudiantes de la Universidad de Caldas. Manizales, Colombia: Universidad de Caldas.

El Tiempo. (21 de julio de 2013). Colombia, el país con más uniones libres de América. Recuperado de http://www.eltiempo.com/archivo/documento/CMS-12940493.

Flórez, C.E. y Sánchez, L.M. (2013). Fecundidad y familia en Colombia: ¿hacia una segunda transición demográfica? Bogotá, Colombia: PROFAMILIA.

Fraser, N. (2015). Fortunas del feminismo: del capitalismo gestionado por el Estado a la crisis neoliberal. Madrid, España: IAEN.

Giddens, A. (2000). Sociología. Ciudad de México, México: Alianza editorial.

Giddens, A. (2007). Un mundo desbocado, los efectos de la globalización en nuestras vidas. Ciudad de México, México: Taurus.

González, R.L. y Restrepo, P.J. (2010). Prácticas de continuidad de los vínculos parentales en las familias transnacionales colombianas en España (Comunidad valenciana, España, Eje Cafetero, Colombia). Revista Latinoamericana de Estudios de Familia, 2, 79-97.

Gutiérrez de Pineda, V. (1994). La familia y cultura en Colombia: tipologías, funciones y dinámico de lo familia. Medellín, Colombia: Universidad de Antioquia.

Guzmán, R.D. y Chaparro, G.N. (2013). Restitución de tierras y enfoque de género. Bogotá, Colombia: Dejusticia.

Herrera, M.F. (2015). Políticas de vida, prácticas de sí, en los contextos de fragmentación institucional. Revista Latinoamericana de Estudios de Familia, 7, 80-96.

Jelin, E. (2010). Pan y afectos: la transformación de las familias. Buenos Aires, Argentina: Fondo de Cultura Económica.

Jurado, C. y Tobasura, I. (2012). Dilema de la juventud en territorios rurales de Colombia. 
¿Campo o ciudad? Revista Latinoamericana de Ciencias Sociales, Niñez y Juventud, 10 (1), 63-77.

López, L.M. y Loaiza, M.O. (2009). Padres o madres migrantes internacionales y su familia: oportunidades y nuevos desafíos. Revista Latinoamericana de Ciencias Sociales, Niñez y Juventud, 7 (2), 837-860.

Mateos, S. (2013). Construcción de la feminidad normativa y sujeto político. Investigaciones Feministas: Papeles de Estudios de Mujeres, Feministas y de Género, 4, 297-321.

Ministerio de Cultura. (2011). Paisaje cultural cafetero: un paisaje cultural productivo en permanente desarrollo. Bogotá, Colombia: Ministerio de Cultura.

Pachón, X. (2007). La familia en Colombia a lo largo del siglo XX. En Puyana, Y. y Ramírez, M.H. (ed.), Familias, cambios y estrategias (pp. 145-160). Bogotá, Colombia: Universidad Nacional, Alcaldía Mayor de Bogotá.

Palacio, M.C. (2010). Los tiempos familiares en la sociedad contemporánea: la trayectoria de una configuración. Revista Latinoamericana de Estudios de Familia, 2, 9-30.

Palacio, V.M. (2009). Los cambios y transformaciones en la familia: una paradoja entre lo sólido y lo líquido. Revista Latinoamericana de Estudios de Familia, 1, 46-60.

Parella, S. (2007). Los vínculos afectivos y de cuidado en las familias transnacionales. Migrantes ecuatorianos y peruanos en España. Migraciones Internacionales, 4 (2), 151-188.

Puyana, V.Y. (2012). Las políticas de familia en Colombia entre la orientación asistencial y la democrática. Revista Latinoamericana de Estudios de Familia, 4, 210-226.

Restrepo, J.E. (2016). Cambios y permanencias en las familias con padres migrantes retornados a Antioquia y el Eje Cafetero colombiano. Migraciones, 40, 127-152.

Robledo, S.C. (2008). Imaginarios regionales del Eje Cafetero de Colombia: paisaje de paisajes. Tijuana, México: Colegio de la Frontera Norte.

Rojas, G.C. (2013). Transiciones juveniles y nuevas configuraciones familiares en Manizales, Colombia. Revista Latinoamericana de Estudios de Familia, 5, 63-80.

Sandoval, M.L. y Moreno, C. (2008). Virginia Gutiérrez de Pineda: aportes al desarrollo del pensamiento social del conocimiento de la familia y la formación de nación en Colombia. Revista de Antropología y Sociología: VIRAJES, 10, 107-154.

Silva Arias, A.C. y González, P. (2007). Una revisión a la composición y transición demográfica en Colombia. Revista Facultad de Ciencias Económicas: Investigación y Reflexión, XV (2), 6178.

Soto, C.D. (2011). La migración internacional paterna o materna: una lectura desde los sujetos jóvenes. Revista Latinoamericana de Ciencias Sociales, Niñez y Juventud, 10 (1), 611-624.

Therborn, G. (2007). Familias en el mundo. Historia y futuro en el umbral del siglo XXI. En Arriagada, I. (ed.), Familia y políticas públicas en América Latina una historia de desencuentros. Santiago de Chile, Chile: CEPAL.

Zapata, A. (2009). Familia transnacional y remesas: padres y madres migrantes. Revista Latinoamericana de Ciencias Sociales, Niñez y Juventud, 7 (2), 1749-1769.

Zuleta, E. (2010). Tres culturas, tres familias, y otros ensayos. Medellín, Colombia: Hombre nuevo editores. 